\title{
IoT and Big Data Analytics for Smart Buildings: A Survey
}

\author{
A Daissaoui a,b*, A Boulmakoul ${ }^{\text {c }}$ L Karim ${ }^{\text {d }}$, A Lbath ${ }^{\text {a }}$ \\ ${ }^{a}$ LIG/ MRIM, CNRS, University Grenoble Alpes, France \\ ${ }^{b}$ EMSI, LPRI, Casablanca, Morocco \\ ${ }^{c}$ LIM/IOS., FSTM, Hassan II University of Casablanca, Morocco \\ ${ }^{d}$ ENSA Berrechid, Hassan 1st University, Morocco
}

\begin{abstract}
The processes of digital transformation have involved a variety of socio-technical activities, with the objective of increasing productivity, safety and quality of execution, sustainable development, collaborative working, and solutions for the sustainable smart buildings. The major technological trends have changed the building sector and revealing new understanding on how data generated by all these new technologies can be used to achieve the essence of smart building objective. Current smart building management systems incorporate a variety of IoT technologies, a structured data storage environment and analytics data tools. Their objectives are to observe the condition of specific areas and apply appropriate rules to preserve or improve comfort while saving energy. In this paper, we propose a review of related works to IoT, Big Data analytics in smart buildings.
\end{abstract}

Keywords: Smart buildings, IoT, Big data analytics, Reactif systems, Complex event processing

\section{Introduction}

The growing interest in smart Buildings, and the emergence of new technologies in this sector, has given rise to several studies with the objective of implementing different types of applications such as energy optimization, simplification of building management, improvement of residents comfort, reactive alarm management, personal protection, assets protection, intrusion event management, etc. The increase in security vulnerabilities resulting from the interaction between cyber and physical entities has the effect to enhance this research focus. Intelligent management systems proposed allow the generation of relevant characteristics, topological visualization for the security of a building, and verification of access control policies to meet security obligations [30]. Recent research has proposed to describe uniform metadata for modelling buildings. These practices use sensor ontologies, subsystems, and relationships, ensuring interoperable and portable [9] [18] [22] applications. At present, we cannot talk about smart buildings without mentioning two inseparable components, namely the Internet of Things (IoT), which is made of all the connected sensors and the storage environment for the data generated by these sensors [10]. IoT has become the key technological element in smart Buildings, any modern construction designed to be intelligent must necessarily integrate connected objects.
It is therefore important to understand the integration of the IoT in this kind of construction also to provide managerial tools in

order to make them as dynamic as possible and to allow the most enjoyable experience possible for its occupants.

Furthermore, we cannot attribute SMART qualification to any building without analyzing the data generated from IoT objects that are in. For each facility looked, choosing an appropriate analytical method and proceed with Data selection, data cleaning, and data preparation are the first steps in the process of analysis. Nevertheless, storing and searching massive data in real time is a laborious task for smart Buildings.

The main objective of this work is to review the current literature of smart buildings through a focus on IoT and Big Data, which are the two big technological components in our context. This paper is structured as follows: section 1 introduces the concept of smart Buildings and related technologies. Section 2 focuses on the field of the Internet of Things, the architecture, the technologies, and the applications. Section 3 present techniques used in bigdata analytics ecosystem. Section 4 present Indoor technology for locating people and object in building, Finally, Section 5 concludes the paper.

\footnotetext{
${ }^{*}$ Corresponding author. Tel.: +1234567890

Fax: +9876543210; E-mail: Author.Name@iasks.org

(C) 2020 International Association for Sharing Knowledge and Sustainability.

DOI: $10.5383 / J U S P N .13 .01 .004$
} 


\section{IoT in Smart Buildings}

Smart building is a central element in improving cities and infrastructures, but above all in improving the comfort of residents. It allows for better energy efficiency, controls safety aspects, and provides a better framework for comfort, quality of life and services at the request of residents and businesses. Several definitions are proposed, most often focused on the energy aspect and linked to the concept of "smart grid". When a building integrates intelligent management systems, massive data storage and analytics to facilitate and improve energy management, electrical devices on the grid that learn habits and adapt to behaviors, considered as an smart Building system. Thus, the management of equipment and services is intelligent through the introduction of IoT and Big Data technologies and using data in analytics and automatic learning. In a generic way, an intelligent system in the context of an smart Building is composed of three levels:

- The infrastructure level of the input data: represents all the data sources generated by the connected objects in the building such as energy consumption, humidity level, indoor and outdoor temperature, number of alarm activation and deactivation, etc..

- $\quad$ System infrastructure level: represents the core of the intelligent system since it allows the collection, processing, and merging and storage data in a NoSQL database. Thus, this allows the use of this data for knowledge extraction through data mining algorithms, automatic learning through artificial intelligence algorithms or simply offering reporting services [19].

- The level of services: represents the list of services offered by the system to building managers, residents and energy suppliers, etc...

\subsection{Technology and architecture}

The beating heart of smart Buildings is the set of technological components based on IoT, allowing control and data generation for user services [42]. Heating, Ventilation and Air-Conditioning (HVAC) provides the smart Building with enough heating, adequate ventilation and a better air conditioning system [38].

Smart Building is a new technology that is worth a modern building architecture from planning to implementation through the implementation of IoT. Integration of IoT in smart Buildings has the benefit of delivering both real-time measurements and contextual information. Activities such as lighting, vehicle parking, use of household appliances, access authorization can be recognized by using connected objects.

In addition, real-time location (all places visited by users) by GPS is now possible. In addition, mobile applications will help to collect other personal data such as age, gender or weight. All collected data can be managed by an advanced database server, and within a large data management framework to predict trends. Among these supporting technologies, one of the trends is the development of the Internet of Things (IoT), because one of the challenges of smart Buildings is to deal with a complex network of interconnected functional entities in different aspects of a building [41]. With the use of IoT, there is enormous potential for considerable progress towards the achievement of envisaged objectives. Considering the diversity of IoT stakeholders and applications, a variety of definitions of technology are available in the literature [21].

From a technological point of view, IoT can be achieved by the alignment of three main approaches, namely: Objects oriented vision, Internet oriented vision and semantic oriented vision [28].

The IoT architecture is designed to provide all objects with identification, detection, networking and processing capabilities, so that they can exchange and share information with each other and develop advanced services over the Internet. In this way, interconnection would facilitate a more complete understanding of complex systems; provide contextsensitive dynamic decision-making capabilities and intelligence autonomy. These capabilities open the way to achieving the objectives in smart Buildings, namely integrated ambient intelligence by providing a holistic network that supports ubiquitous computing [15] and awareness of the rising context among devices [8].

The implications of IoT are twofold:

- Integration of detection, storage, networking, processing and computing capabilities into everyday objects and their implementation online, even if they were not initially designed with these capabilities.

- Integration of networks that include the abovementioned objects. This would make them accessible via the network. The ambient intelligence provided by IoT allows each object to understand its environment, establish meaningful interaction with people and help them make decisions. Although researchers still face technical challenges in developing, applying and eventually maturing IoT [32], it is intended that the technology will be applicable to a variety of industries, such as health, manufacturing, retail, agriculture, industrial automation, etc.[27].

As a recent trend, IoT has begun to enter the construction industry in recent years. Researchers and practitioners are exploring the benefits and inconveniences of IoT through its effective implementation. For example, several companies, including IBM and Intel, are now launching their smart Building products around the world [2], demonstrating the competitive advantage and future trend of IoT.

However, to the authors' knowledge, although there are surveys on smart Buildings based on IoT [10], the current literature lacks a comprehensive review and analysis of IoT applications in the general areas of future building development. The most used architecture of IoT is the three-layer architecture, namely perception layer, network layer and application layer.

The perception layer includes perception nodes and perception networks, which integrated into the target and is responsible for detection and data collection. The network layer is responsible for data transport, which is the most important layer of the 


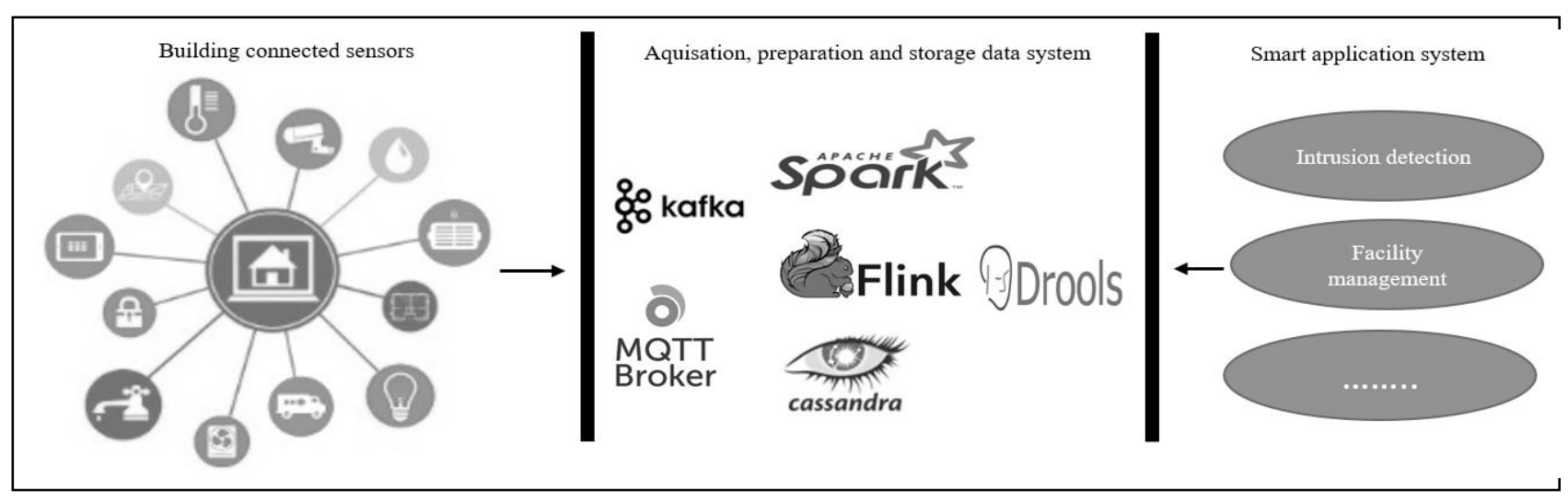

Fig. 1. Basic smart building architecture

architecture, because it is the convergence of various devices and the communication infrastructure. Finally, application layer is the top layer where end users interact. It receives the transmitted data and deliver it to users for other services.

Figure 1 represents the layers of the architecture that can handle the smart building system from data generation by the connected objects that are installed in the building until the application that will be used by end users, it gives also a overview of typical recent technology to handle data processing.

\subsection{LPWAN Technologies}

The sensors are usually located in areas where batteries can only power them; this demands a low power consuming communication protocol. The widely employed traditional short-range communication technologies cannot be deployed for WSN networks. Further, solutions based on mobile cellular communications (e.g., 2G, 3G, and 4G) could ensure a more extensive transmission range. However, it depletes device's energy. Therefore, IoT applications requirement leads to the emergence of Low Power Wide Area Network (LPWAN)[25]. Unlike other radio communication technologies like Zigbee that use mesh networks, LPWA networks employ star network topology. The deployment of mesh networks to connect a huge number of sensors that are physically dispersed in a wide area is very expensive. Moreover, as data is transmitted through multi hops towards a gateway, some devices get more congested than others due to an increase in network traffic, which reduces their batteries lifetime (i.e., excessive energy consumption) and thus limit the entire network lifetime [14]. LoRa, Sigfox, and NB-IoT can easily overcome the cost of deployment as these employ star topology.

\subsection{Applications and opportunities}

The use of IoT in smart buildings will generate several applications, like the following:

Facilities Management: In the life cycle of a building, the operating phase takes the longest time. Therefore, facilities management is another objective of smart Buildings, which integrates organizational activities to maintain effective and efficient services. Facilities Management (FM) requires timely preventive maintenance and detection of building equipment defects to ensure the optimal condition of the facility. Traditional FM has problems with lower data quality, longer
Under these conditions, IoT provides adaptive and real-time access to building facilities for the staff concerned. An effective FM will bring many benefits, including the possibility of improving the health and comfort of occupants, improving the overall quality of installation services, reducing the cost of repairs and energy consumption of buildings, providing the means for efficient planning and use of resources, etc. [1]. Several use cases of IoT in intelligent plant management are available. A D'Elia et al [5] smart Building maintenance platform developed a set of contextual intelligent maintenance applications that used environmental sensors to monitor related variables, such as temperature and humidity, and automatically report feedback. For example, if an "out of range temperature", a message is generated; this platform is able to detect the location and possible defective equipment, in this case the HVAC system, and to alert the corresponding human operator via a personal device. In addition, an example of a suggested corrective intervention will be provided to staff and tenant to facilitate the repair process. The idea of the platform could be extended to all smart Building construction systems, as well as to the building structure for automatic monitoring of the entire building. An acoustic HVAC system developed by collecting, transmitting audio signals over the Internet then processing and characterizing the audio signature with corresponding data from the HVAC system components proposed in [35][39].

The fact is that intelligent facility management IoT-based has been applied by industrial companies. Energy management: Energy efficiency in buildings is one of the most important research topics to date, and not only in the development of smart Buildings, since buildings represent $40 \%$ of the world's total energy consumption. On the other hand, an smart Building must not compromise the level of service for users or occupants of the building in order to achieve this objective, which requires a solution that give satisfaction to all parties. Energy management systems already exist in commercial buildings [3] that control, monitor and optimize the current energy consumption of buildings. These systems usually install non-intrusive meters on electrical circuits to collect energy consumption data for users and building manager.

However, the potential for improvement is still very significant at this stage. Smart Buildings require customization according to specific requirements, which requires a certain level of contextual knowledge. This means that the state of the environment and occupants plays a crucial role in the operation of an smart Building. For illustration, HVAC system need adjustment according to the number of people in the room and 
the lighting system must monitor the light intensity outside the building and adjust the lighting inside accordingly. IoT helps the smart grid to manage energy consumption from a macroeconomic perspective with regard to the energy efficiency of buildings. The smart grid consists of computer networks that work with the electrical infrastructure to manage and monitor energy consumption. Traditional electricity grids are unable to meet the growing and fluctuating demand for energy. The smart grid establishes a two-way communication between the electricity supplier and the customer that allows both information and energy transfer. The key elements of the smart grid that allows bidirectional communication is the smart meter.

A smart meter is an advanced energy meter that not only measures energy consumption in real time, but also other properties such as voltage, phase angle and frequency. In addition, theycan also communicate diagnostic information and communicate with other smart meters [34]. The energy efficiency in buildings will become increasingly important as industry around the world sets targets to promote this task.

Occupants and resources location tracking: The location inside is of great value for improving building performance. For example, occupants who are not familiar with a building could have access to navigation to their destination; occupancy information acquired from location information could be used to distribute the resource in a balanced manner; building managers could find any equipment or facilities that require maintenance or repairs to increase their productivity at work. In addition, occupant location will help to understand occupant behaviors and predict unique events within buildings. At this time, the role of the building occupants wasn't sufficiently considered into account and the behavior of the occupants is supposed to be static, and in some cases, one or more representative profiles are used for building operation and management [31]. This has consequences such as inefficient use of resources or waste of energy. Understanding the behavior of occupants is complicated; while the ability to locate them when they are inside buildings is a major challenge. Clearly, current GPS technologies do not have the desired accuracy inside buildings, they are primarily designed for geo-fencing and area-based services. A promising part of IoT is the improvement of microlocation technologies that allow any entity to be located with very high accuracy possibly up to a few centimeters. In essence, micro location is a geo-fencing with a high degree of certainty, which allows any object within the building to be positioned and tracked and, therefore, is used for better and more efficient service delivery. Five main technologies on the IoT based micro location in market [17], namely: Bluetooth Low-Energy (BLE)-Based Beacons/iBeacons, UWBBased Micro-location (Ultra-wide band), and Wireless positioning systems, Magnetic field mapping, and RFID.

Enhancement of interior comfort: Like energy efficiency, occupant comfort is another major concern of smart Buildings in that, the fundamental requirement of buildings is to maintain comfort living conditions for building occupants. In addition, people spend on average $80 \%$ of their lives in buildings [36], so a healthy and comfortable indoor environment is important for the well-being and productivity of occupants.

Robust monitoring and control of the indoor built environment is necessary in real time. In advanced level, future smart building systems, such as HVAC, will integer sensors and actuators, so that temperature settings will according to occupants' preferences and needs based on historical information through empirical learning automatically configured. The development of these intelligent functions is an IoT system based, for data collection, decision making, sending orders, etc. The smart house is generally the first type of building to start with research on occupant comfort using an IoT system. Kelly et al [40] implemented an IoT system for monitoring indoor environmental conditions and the use of utilities in residential buildings. Other IoT applications studied in the literature such as occupant safety and health security, effective resource management for convenience, etc [7][23].

Notwithstanding the benefits and progress brought by IoT to the construction industry, some challenges and problems remain for researchers in different fields such as security and confidentiality, data acquisition, processing and storage issues, but also feasibility, adaptability and practicability in a complete ecosystem.

\section{Big data and smart buildings}

In the smart Building context, a huge amount of data created every second and reaches critical sizes. For this purpose, several solutions proposed for large-scale data processing. The market-leading solutions are Spark and Hadoop [29]. Spark and Hadoop are both large data frames, but they do not really have the same use. MapReduce's operating mode may be sufficient if the operational and reporting requirements are essentially static and it is possible to wait until the batch processing is complete. However, if we need to analyze data in streaming, as is the case for processing sensor data in an smart Building, or if applications require a succession of operations, we will probably need to use Spark. This is the case with most of machine learning algorithms, which require several operations. Spark is perfectly adapted to this context. Knowledge extraction from data known as the process of analyzing different types of data to extract models and knowledge using different data mining tools. Many applications use data mining in their implementation such as health care data analysis, education system, production control, decision-making, etc...
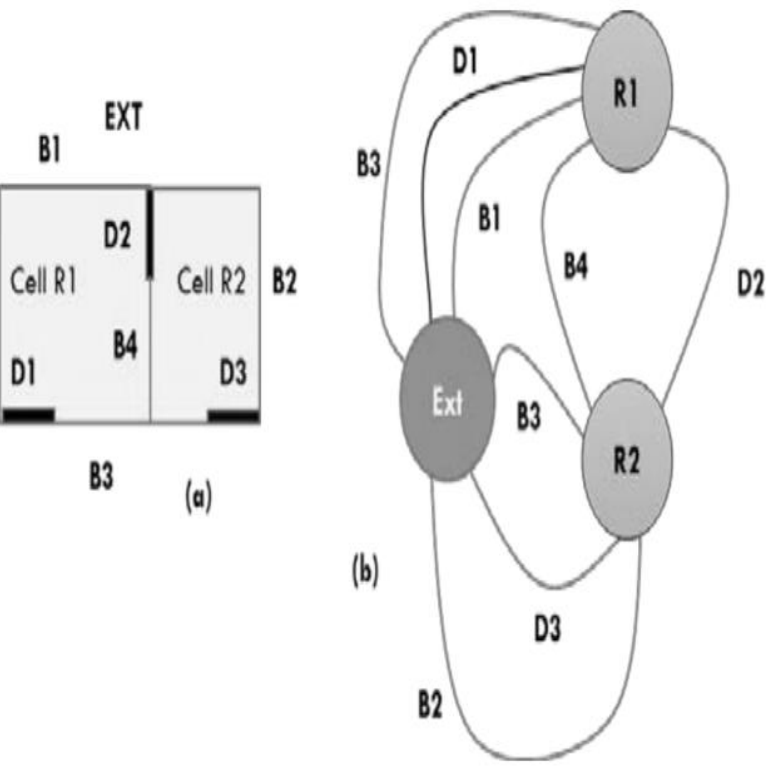

Fig. 2 (a) Topographic space, (b) Adjacency Graph. 


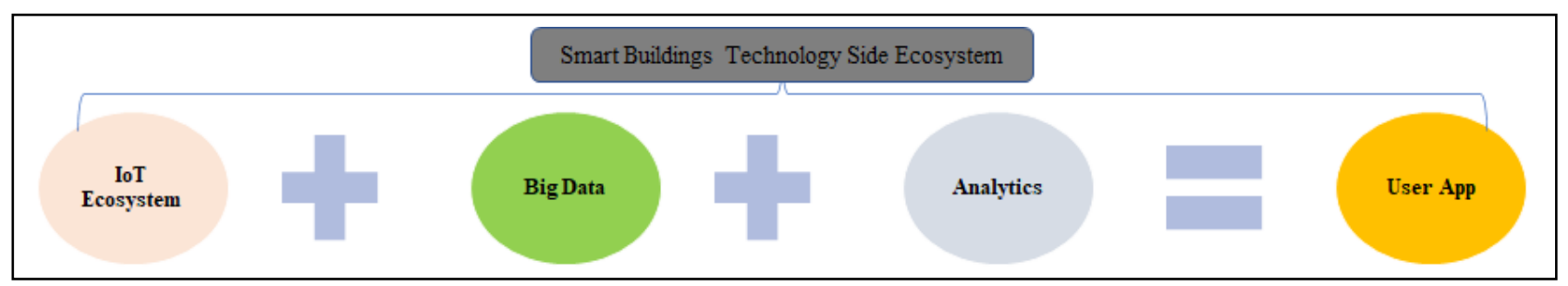

Fig. 3. Relation between smart building components

Different clustering algorithms exist such as K-means algorithm, hierarchical grouping algorithm, self-organizing card algorithm and expectation maximizing grouping algorithm. The choice of using a specific algorithm over another depends on several parameters: the size of the data set, number of clusters, type of data set, etc. The analysis of this voluminous data makes it possible to achieve the global vision of smart Buildings to simplify building management, reduce energy consumption, secure property and people, and provide a simplified living environment. In this part of section, we provide a brief description of some popular algorithms used to explore data generated and stored from smart building.

\subsection{Probabilistic graphical model}

A structure of conditional algorithms that try to learn the dependencies between the variables for which the data collected from the sensors. Generally consists of two parts: the first part is learning the structure of variable dependency, which is an essential step in Bayesian networks. The second part consists in learning the conditional probability distributions for the related variables in the graph. This is an essential element in almost all types of graphical models frequently used to calculate the probability a space occupation. A stochastic graphical model based on stochastic agents to simulate building occupancy proposed in [12]. The graphical model was to capture information on the average occupancy of the different areas of a building as well as the correlation of occupancy between the different areas.

Stoppel et al [13] have integrated probabilistic methods into building energy simulation models to optimize construction. Chen et al [43] propose non-homogeneous Markov chain models to model regular occupancy in commercial buildings in single-occupancy and multi-occupancy multi-zone areas respectively. Lam et al [26] achieved the following objectives of $80 \%$ accuracy in occupancy detection using hidden Markov models (HMM). The HMM method explicitly models the time correlations between environmental variables and consecutive time step occupancy levels and is therefore a powerful prediction method.

\subsection{System identification techniques}

System identification techniques involve the use of statistical methods to construct mathematical models of dynamic system models. Many algorithms of this class in the field of building occupancy estimation attempt to find building occupancy profiles using the following statistical techniques.

Given a particular set of geometric and physical characteristics of the building, disturbances, and the number of occupants on one side and other monitored variables on the other side. Technical identification systems used to find the best dynamic identification techniques that link the two sides. In [6] an approach followed to form a model of occupancy within a building. During the inference, however, the authors use the estimated model and exiting observation to find the number of occupants. In [11], Duarte et al. detail occupancy diversity factors for several locations within a building, such as offices, break rooms, corridors, conference rooms and washrooms.

The final objective was to provide information to facilitate building management and energy optimization as well as assistance in improving security in the context of intrusion.

\subsection{Vector support machines}

Support vector machines (SVMs) belong to the supervised learning algorithm class. Their objective is to predict the state of building in context facility management, comfort of resident, and security by estimate the occupancy rate or zone. The SVM algorithms attempt to find the maximum margin hyperplane that can separate the data into two categories for occupied and empty state. Pair data that are not linearly separable, can be modeling to larger dimensions using non-linear kernel functions such as radial basic functions and use appropriately for classification. In [20], Shih use SVM to count and track people both day and night. The authors applied the SVM to estimate the observation density, based on the probability (similarity) between the defined target model and the characteristic vector obtained in the test case. Akbar et al [4] use three variants of VCMs: those with RBF cores, those with linear cores, and those with polynomial cores in their work on contextual detection of office occupancy using smart meter data.

\subsection{Data mining and clustering}

There is a considerable documentation on unsupervised learning methods to extract meaningful information on trends in a building. Chen et al [16] explore non-intrusive occupancy monitoring (NIOM) using a clustering approach, followed by thresholding over three metrics: mean power, standard deviation, and power range collected from electricity meter data. Doca et al [37] propose a mining framework, mainly involving rule induction. In [24], Zhao et al. use data mining to learn the behavior of building occupants. Nguyen et al [33] use a categorical non-parametric clustering approach for an unsupervised learning to extract high use areas locations.

In general, to date, several methods and algorithms used in the smart building context. These include statistical and conventional learning methods, neural network-based methods, 
probabilistic graphical modelling, and data mining algorithms. The evaluation and performance of these techniques depend on the purpose and quality of the data used.

\section{Indoor localization application}

Analysis of the behavior or navigation of people in smart buildings has progressed rapidly in recent years. Applications conventionally focus more on the visualization and monitoring of building occupancy and the monitoring of passengers traffic. Various services are provided to building managers and users. These services go a long way toward lighter asset management achieving cost reductions, improving the user experience, and supporting sustainability efforts. Detecting the occupation of space distinguishes two categories. The first uses practices that require smartphone-like geolocation equipment.

The other category, detection is based on a passive approach based on surveillance of areas or spaces instead of identifying devices using cameras Data from the detection of people in spaces is used to generate dynamic classifications. Precisely these data are spatio-temporal and machine-learning algorithms allow producing several clustering according to homogeneous periods.

\subsection{Indoor Objects in IndoorGML}

The OGC® IndoorGML standard proposes to establish a common scheme for indoor navigation applications. It models topology and semantics of indoor spaces as shown in figure 2 It defines the following information on indoor space: (1) Context and navigation constraints, (2) Spatial subdivisions and types of connectivity between spaces, (3) Geometric and semantic properties of spaces and connectivity, (4) Navigation networks (logical and metric) and their relationships. The difference between indoor and outdoor space is that an interior space is composed of complex constraints such as corridors, doors, stairs, elevators, etc.

\subsection{Indoor Location Technology}

Depending on the required accuracy (granularity) of the positioning, solutions with different locating methods are available, which can also be based on different technologies. Each with different advantages and disadvantages. Especially solutions based on WLAN, the so-called fingerprinting, and the well-known GPS have not been able to establish themselves in practice.

Although WLAN is now available in most cases, it is often not stable enough and the setup of a positioning system requires a lot of effort. GPS is ideally suited for outdoor areas. However, GPS signals do not achieve the necessary penetration in buildings. RFID systems are cheap, but only a static position can be determined. It is not possible with these technologies to track the free movement of a worker or a pallet in a building.

Bluetooth Low Energy and Beacons have emerged as the ideal technology for these requirements. In the meantime, modern AoA locating procedures with Bluetooth based systems can locate accuracy in the sub-meter range. Up to now, systems based on ultrawideband technology have achieved the highest accuracy. Since, in addition to the granularity of the position determination, the price of a system plays a major role in the decision for a certain technology, and UWB is by far the most expensive technology, BLE now also has great advantages over UWB.

\subsubsection{UWB - Ultra-Wideband}

UWB enables very accurate three-dimensional positioning, which has to be paid for with high acquisition costs and high effort during system setup. A continuous asset tracking can be realized with UWB.

\subsubsection{RFID - Radio Frequency Identification}

With the help of so-called RFID tags, RFID provides a static position indication with very inexpensive hardware. A continuous asset tracking is not possible, but only information about whether an asset was registered at a certain moment at a certain location.

\subsubsection{BLE/RSSI}

Bluetooth low energy technology enables continuous asset tracking with at least room-accurate location. RSSI refers to the method of indoor tracking using beacons and means Received Signal Strength Indicator, which is used as the main indicator of the position calculation via beacons.

\subsubsection{BLE/AoA}

This is also indoor tracking via Bluetooth Low Energy sensors/beacons. The position is calculated with another method, using the Angle of Arrival. This calculation allows much more precise localizations but is accompanied by increased effort in setting up the sensor infrastructure and slightly higher hardware costs. Nevertheless, the costs remain below a comparable UWB system.

\subsubsection{WLAN}

Asset tracking and positioning via the existing WLAN network has not proven itself in practice, neither in terms of location accuracy nor in terms of system stability. In addition, the bandwidth is extremely strained by the localization.

\section{Conclusion}

This paper presents a state of the art on research for the smart building's development. First, we have discussed the use of IoT that offers new opportunities for smart Building management.

The large data volume gathered from sensor networks feeds Big Data databases and opens the deep analytical dimension for identifying models.

In general, to build a smart building system application for any end of use, we need four components, (1) sensors with IoT communication technology, (2) Networking witch allow to communicate between building sensors and cloud system where a backend configuration work to process data, (3) Bigdata technology to store the huge amount of data gathered and finally (4) a analytic process algorithm to extract knowledge. Figure 3 resume the relation between all this four elements and user application. 
Based smart Building operators' needs and also the progressing interest of indoor localization, in our future work, we propose the deployment of sensor data integration and intelligent services ecosystem for the dynamic control of the movement of people in the spaces of a smart building based on fusion of multiple indoor technology and using fuzzy clustering mining to extract knowledge.

\section{References}

[1] (2017) "Facility management and smart buildings: smart data, insights and integration." https://www.i-scoop.eu/internet-of-thingsguide/facility-management-iot-smartbuildings.

[2] (2018) "Watson Internet of Things." https://www.ibm.com/internetof-things/spotlight/iotzones/iot-buildings.

[3] (2019) "ACISTM Building Energy Management System", http://www.airedale.com/web/Controls/Building-Energy-

ManagementBEMS/ACISTM-Building-EnergyManagementSystem.htm. .

[4] A. Akbar, M. Nati, F. Carrez, K. Moessner, (2015) "Contextual occupancy detection for smart office by pattern recognition of electricity consumption data." in:Communications (ICC), IEEE International Conference on, IEEE: 561-566. https://doi.org/10.1109/ICC.2015.7248381

[5] A. D'Elia, L. Roffia, G. Zamagni, F. Vergari, P. Bellavista, A. Toninelli, S. Mattarozzi, (2010) "Smart applications for the maintenance of large buildings: how to achieve ontologybased interoperability at the information level." Computers and Communications (ISCC), IEEE Symposium on: 1077-1082. https://doi.org/10.1109/ISCC.2010.5546639

[6] A. Ebadat, G. Bottegal, D. Varagnolo, B. Wahlberg, K.H Johansson, (2013) "Estimation of building occupancy levels through environmental signals deconvolution." Proceedings of the 5th ACM Workshop on Embedded Systems For Energy-Efficient Buildings: 1-8. https://doi.org/10.1145/2528282.2528290

[7] A. Piscitello, F. Paduano, A.A. Nacci, D. Noferi, M.D. Santambrogio, D. Sciuto, (2015) "Danger-system: exploring new ways to manage, occupants safety in smart building." IEEE 2nd World Forum on Internet of Things (WF-IoT): 675-680. https://doi.org/10.1109/WF-IoT.2015.7389135

[8] A. Whitmore, A. Agarwal, L. Da Xu, (2015) "The internet of things - a survey of topics and trends." Inf. Syst. Front. 17(2):261-274. https://doi.org/10.1007/s10796-014-9489-2

[9] B. Balaji, A. Bhattacharya, G. Fierro, J. Gao, J. Gluck, D. Hong et al, (2016) "Towards a unified metadata schema for buildings." Proceedings of the 3rd ACM international conference on systems for energy-efficient built environments. ACM: 41-50. https://doi.org/10.1145/2993422.2993577

[10] B.L.R. Stojkoska, K.V. Trivodaliev (2017) "A review of Internet of Things for smart home: challenges and solutions" J. Clean. Prod. 140: 1454-1464. https://doi.org/10.1016/j.jclepro.2016.10.006

[11] C. Duarte, K. Van Den Wymelenberg, C. Rieger, (2013) "Revealing occupancy patterns in an office building through the use of occupancy sensor data." Energy Build: 587-595. https://doi.org/10.1016/j.enbuild.2013.08.062

[12] C. Liao, Y. Lin, P. Barooah, (2012) "Agent-based and graphical modelling of building occupancy." J. Build. Perform. Simul. 5(1):5-25 https://doi.org/10.1080/19401493.2010.531143

[13] C.M. Stoppel, F. Leite, (2014) "Integrating probabilistic methods for describing occupant presence with building energy simulation models." $\quad$ Energy $\quad$ Build. $\quad 68: \quad 99-107$. https://doi.org/10.1016/j.enbuild.2013.08.042

[14] C.V. Ravikumar, B. Kalapraveen, ( 2016) "Robust Neural Network based multiuser detector in MC-CDMA for multiple access mitigation", Indian Journal of Science \& Technology. Vol 9, issue 30, https://doi.org/10.17485/ijst/2016/v9i30/95994

[15] D. Bandyopadhyay, J. Sen, (2011) "Internet of things: applications and challenges in technology and standardization." Wirel. Pers. Commun. 58(1): 49-69. https://doi.org/10.1007/s11277-011-0288-5

[16] D. Chen, S. Barker, A. Subbaswamy, D. Irwin, P. Shenoy, (2013) "Non-intrusive occupancy monitoring using smart meters." in: Proceedings of the 5th ACM Workshop on Embedded Systems For Energy-Efficient Buildings: 1-8. https://doi.org/10.1145/2528282.2528294

[17] F. Zafari, I. Papapanagiotou, K. Christidis, (2016) "Microlocation for internet-of-things equipped smart buildings." IEEE Internet Things J.3 (1): 96-112 https://doi.org/10.1109/JIOT.2015.2442956

[18] G. Fierro, Culler DE. Xbos (2015) "An extensible building operating system." Proceedings of the 2nd ACM international conference on embedded systems for energy-efficient built environments. ACM;119-20.

[19] H. Asri, H. Mousannif, H. Al Moatassime, (2019) "Reality mining and predictive analytics for building smart applications." Journal ofBig Data, 6: 66. https://doi.org/10.1186/s40537-019-0227-y

[20] H.-C. Shih, (2014) "A robust occupancy detection and tracking algorithm for the automatic monitoring and commissioning of a $\begin{array}{llll}\text { building." } & \text { Energy } & \text { Build. } & \text { 77: }\end{array}$ https://doi.org/10.1016/j.enbuild.2014.03.069

[21] J. Gubbi, R. Buyya, S. Marusic, M. Palaniswami, (2013) "Internet of Things (IoT): a vision, architectural elements, and future directions." Futur. Gener. Comput. Syst: 1645-1660. https://doi.org/10.1016/j.future.2013.01.010

[22] J. Mengda et al, (2019) "Automation in Construction" 101:111126 https://doi.org/10.1016/j.autcon.2019.01.023

[23] J. Patel, G. Panchal, (2018) "An IoT-based portable smart meeting space with real-time room occupancy." Intelligent Communication and Computational Technologies, Springer, Singapore: 35-42. https://doi.org/10.1007/978-981-10-5523-2_4

[24] J. Zhao, B. Lasternas, K.P. Lam, R. Yun, V. Loftness, (2014) "Occupant behavior and schedule modeling for building energy simulation through office appliance power consumption data mining $\mu$." EnergyBuild.82:341-355.

https://doi.org/10.1016/j.enbuild.2014.07.033

[25] K. Mekki, E. Bajic, F. Chaxel, F. Meyer, (2018) "comparative study of LPWAN technologies for large-scale IoT deployment", ICT Express. https://doi.org/10.1016/j.icte.2017.12.005

[26] K.P. Lam, M. Höynck, B. Dong, B. Andrews, Y.-S. Chiou, R. Zhang, D. Benitez, J. Choi, et al., (2009) "Occupancy detection through an extensive environmental sensor network in an open-plan office building." IBPSA Build. Simul. 145: 1452-1459.

[27] Knud Lasse Lueth, (2015) "The 10 most popular Internet of Things applications right now.",https://iot-analytics.com/10-internetof-thingsapplications.

[28] L. Atzori, A. Iera, G. Morabito, (2010) "The internet of things: a survey." Comput. Netw. 54(15): 2787-2805. https://doi.org/10.1016/j.comnet.2010.05.010

[29] L. Karim, A. Boulmakoul, A. Lbath, (2017) "Real time analytics of urban congestion trajectories on Hadoop-MongoDB cloud ecosystem." the second International Conference on Internet of Things, Data and Cloud Computing (ICC), At Cambridge city, Churchill 
College. University of Cambridge, United Kingdom. ACM Digital Library. $\quad$ ISBN: 978-1-4503-4774-7. https://doi.org/10.1145/3018896.3018923

[30] L. Pasquale, C. Ghezzi, C. Menghi, C. Tsigkanos, and B. Nuseibeh,(2014) "Topology Aware Adaptive Security." Proc. of the 9th ACM Int. Symposium On Software Engineering for Adaptive and Self-Managing Systems: 43-48. https://doi.org/10.1145/2593929.2593939

[31] M. Jia, R.S. Srinivasan, A.A. Raheem, (2017) “from occupancy to occupant behavior: an analytical survey of data acquisition technologies, modeling methodologies and simulation coupling mechanisms for building energy efficiency." Renew. Sust. Energ. Rev. 68: 525-540. https://doi.org/10.1016/j.rser.2016.10.011

[32] M.R. Abdmeziem, D. Tandjaoui, I. Romdhani, (2016) "Architecting the internet of things:state of the art." Robots and Sensor Clouds, Springer, Cham: 55-75. https://doi.org/10.1007/978-3-319$\underline{22168-7 \quad 3}$

[33] N.T. Nguyen, R. Zheng, Z. Han, (2013) “An unsupervised mobile locations extraction approach with incomplete data." in: Wireless Communications and Networking Conference (WCNC): 2119-2124. https://doi.org/10.1109/WCNC.2013.6554890

[34] R. Depuru, L. Wang, V. Devabhaktuni, N. Gudi, (2011) "Smart meters for power grid - challenges, issues, advantages and status." IEEE/PES Power Systems Conference and Exposition: 1-7. https://doi.org/10.1109/PSCE.2011.5772451

[35] R. Srinivasan, M.T. Islam, B. Islam, Z. Wang, T. Sookoor, O Gnawali, S. Nirjon, (2017) "Preventive maintenance of centralized HVAC systems: use of acoustic sensors,feature extraction, and unsupervised learning." Proceedings of the 15th IBPSA Conference: 2518-2524

[36] R. Yang, L. Wang, (2012) "Multi-agent based energy and comfort management in a building environment considering behaviors of occupants." Power and Energy Society General Meeting, IEEE: 1-7. https://doi.org/10.1109/PESGM.2012.6345671

[37] S. D'Oca, T. Hong, (2015) "Occupancy schedules learning process through a data-mining framework." Energy Build. 88: 395408. https://doi.org/10.1016/j.enbuild.2014.11.065

[38] S. Hipwell (2014) “A working model.” International Conference on Intelligent Green Building and Smart Grid (IGBSG): 1-6.

[39] S. Nirjon, R.S. Srinivasan, T. Sookoor, (2017) "SASEM: Smart audio SEnsing based HVAC Maintenance." Chapter Smart Cities: Foundations, Principles and Applications, Wiley, 978-1-119-22639-0.

[40] S.D.T. Kelly, N.K. Suryadevara, S.C. Mukhopadhyay, (2013) "Towards the implementation of IoT for environmental condition monitoring in homes." IEEE Sensors J. 13 (10): 3846-3853. https://doi.org/10.1109/JSEN.2013.2263379

[41] T.K. Hui, R.S. Sherratt, D.D. Sánchez, (2017) "Major requirements for building smart homes in smart cities based on internet of things technologies.” Futur. Gener. Comput. Syst.76: 358-369. https://doi.org/10.1016/j.future.2016.10.026
[42] V. Subbarao, K. Srinivas, R.S. Pavithr, (2019) "A survey of IoT based smart, digital green and intelligent campus." 4th International Conference on Internet of Things: Smart Innovation and Usages (IoTSIU): 1-6. https://doi.org/10.1109/IoT-SIU.2019.8777476

[43] Z. Chen, J. Xu, Y.C.Soh, (2015) "Modeling regular occupancy in commercial buildings using stochastic models." Energy Build. 103: 216-223. https://doi.org/10.1016/j.enbuild.2015.06.009 